\title{
Probabilistic-deterministic approach of controlling the functionality of a technical system in normal and emergency operation modes
}

\author{
Vladimir M. Zababurin*, Marina A. Egorova, and Yuliya A. Polyakova \\ Southern Russian State Polytechnic University (NPI), 346400 Novocherkassk, Russia
}

\begin{abstract}
The main disadvantages of the existing methods of managing the current state of technical systems are revealed. A non-standard approach is proposed for managing the functionality of the system in emergency situations.

The character of the dynamics of the recovery processes of the technical system is determined as its state approaches the emergency one on the basis of the recommendations of the theory of self-organized criticality (SOC). The physical criteria for assessing the current state of the technical system are revealed. The rationale for using the physical indicator of the functional destabilization of the system is given. The signs of the pre-emergency state of the technical system are considered.

A grapho-analytical model for the development of an emergency situation has been developed. The fact of the inevitable increase in the entropy of the system upon its transition to an emergency state is established. Structuring of the system development process in an emergency situation is carried out in three stages.

The methodology for estimating the pre-emergency state of complex open systems is presented. The advantages of the proposed approach to managing the state of technical systems in comparison with traditional ones are established.
\end{abstract}

\section{Actuality and ways of solving the problem of state system management}

The task of effective management of the state of a technical system (hereinafter simply a system) is topical $[1,2]$ and requires an operational solution. At the same time, the key issue is the problem of an objective assessment of the current state of the system in the normal and emergency modes [3]. Modern approaches to analysis and evaluation, based mainly on methods of peer review and intuitive foresight, cannot ensure the receipt of correct information and objective management results. In our opinion, this problem should be solved with probabilistic deterministic positions, including an assessment of the physical

\footnotetext{
* Corresponding author: zababurin64@mail.ru
} 
characteristics of the system, optimization of the level of its functionality, as well as modeling of its possible states under normal and emergency operation.

The key term of the proposed approach is the concept of system functionality. The functionality of the system should be understood as its ability to perform work under specified conditions for a certain time interval [4]. In general, the level of the functionality of the system $(\Phi)$ is determined by the value of its functional $(\varphi)$, which characterizes its current capabilities in performing the basic function related to the total potential $(\mathrm{P})$, the magnitude of which is determined by the limiting values of the given parameter and the ability of the system to perform its functions in full volume.

$$
\Phi=\frac{\varphi}{\mathrm{P}}
$$

It is well known that the dynamics of the states of the system under study should be regarded as a natural result of its metabolic processes with the external environment. On this basis, the energy balance of the system itself and external disturbances of the medium determines the state of the system at a given time interval, the features and the vector of its development on the stability branch and the degree of possible consequences (for the system and the external environment). AT connection with this, the management of the current state of the system is a purposeful influence aimed at minimizing its energy losses and, carried out by analyzing and determining time and physical dynamic characteristics of the system.

\section{Evaluation of the current state of the system}

In practice, the system can operate in the normal operation mode, with the increase of negative external effects, to pass to the pre-emergency state and disintegrate, that is, to go into emergency mode. Important from the point of view of solving practical issues is a reasonable choice of criterion for assessing the current state of the system, objectively operating under any mode of operation of the system. To solve this problem, in our opinion, it is expedient to use the physical parameter of its functional destabilization, which characterizes the energy balance of the interaction of the system Ec and the external environment of the Ev.

Correctly operated system is in a state of energetically adapted equilibrium with the external environment [5]. From these positions, the energy balance of interaction between them can be

$$
E_{C} \approx E_{B . C}
$$

The external environment forms prerequisites for destabilizing the stable operation of the system, causes the growth of entropy and, in the final analysis, its transition to the preemergency state. The complexity of assessing such a state of the system consists in the stochastic nature of the various and interrelated factors that must be taken into account when analyzing the current situation that has arisen.

Mathematically, the dynamics of the functional destabilization index of a system can be represented as a function of a number of input parameters $\mathrm{x} 1, \mathrm{x} 2, \ldots, \mathrm{xn}$ and the time characteristic $\tau$.

$$
\mathrm{K}_{\partial}=\Sigma\left(x_{1}, x_{2}, \ldots, x_{\kappa} ; \tau\right) .
$$

The parameters xi characterize the operating conditions, the state of the elements and other stochastic factors that affect the process of forming the pre-emergency situation. 
In order to improve the efficiency of the estimation and prediction of the pre-emergency state of the system, in our opinion, it is advisable to use the recommendations of the theory of self-organized criticality (SOC) [3]. In accordance with it, there are objectively universal signs of a pre-emergency state of systems.

As the main universal feature of the pre-emergency state of the system, one can use the increase in the time of its reaction and relaxation, since as it approaches the critical transition of the system, it reacts more and more slowly from various influences, it is increasingly difficult to return to its equilibrium point. In particular, the response time to the external environment $\mathrm{rp}$, as well as the recovery rate of working capacity, characterize the ability of the system under investigation to trigger internal protective processes aimed at preserving its organizational structure and increasing its stability. The estimated value of $\tau$ p should be considered a criterion for evaluating the effectiveness of managing the current state of the system, because determines the duration of the normalization of the elements and nodes of the system after negative external influences. The parameter degree of system recoverability and can be determined from expression

$$
\vartheta_{B}=\frac{\Delta \varphi}{\sum \tau_{p}}, \% / \text { hour }
$$

where $\Delta \varphi$ is the specific functionality of the element, $\%$. It is determined by the ratio of the actual performance of the system to the normative, conditioned by the requirements of normative and technical documentation);

$\Sigma$ tp - is the total relaxation time of the element, hour. It is established by means of timekeeping.

The proposed methodological approach and criteria for assessing the current state and conditions for the transition of the system to a pre-emergency and further emergency state using physical laws and conservation laws fundamentally alters the ability of researchers and practitioners to assess the progress of the process compared to, for example, expert judgment based on statistical observations.

\section{Graph-analytical modeling of an emergency situation}

As a rule, the conditions for the formation of an emergency state of the system arise because of the failure to comply with the requirements of the law of the necessary diversity [6]. In our opinion, the study of genesis A specific emergency situation will be more effective if you use graphical-analytical modeling of its dynamics. As is known, any accident from the physical point of view is a quasi-stationary process, including stochastic interrelated events [7]. However, a detailed the characteristics of the system's behavior at various stages of the development of the accident in modern literature do not. To solve this problem, it is proposed to use the grapho-analytical method with the construction of a model of the dynamics of an emergency in a two-dimensional coordinate system, where the time characteristic $(t)$ is used as an argument, and the stability criterion (CG) as a function [8].

Graphical interpretation of the accident as a quasi-stationary process is shown in Fig. 1. Region I is characterized as a zone of sustainable development of the system (passive phase), region II is a zone of catastrophic development (active phase) [9]. 


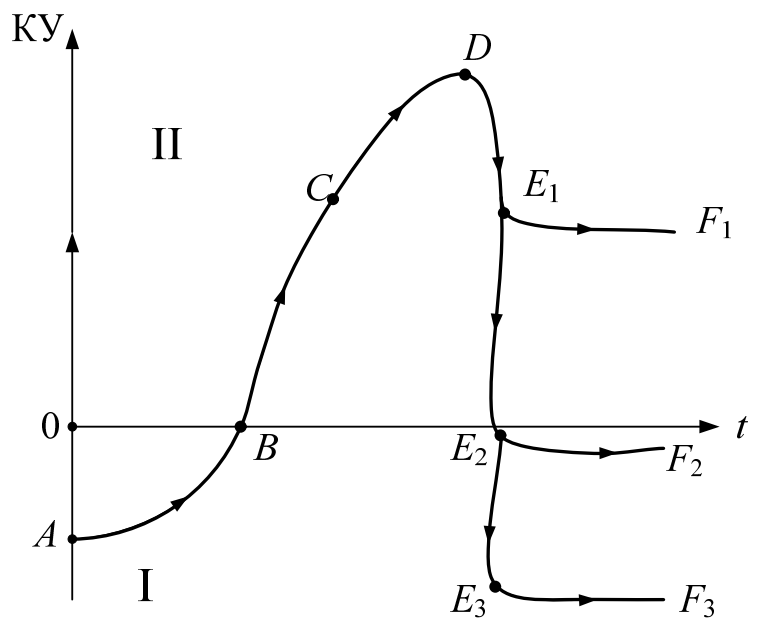

Fig. 1. Dynamics of the development of an emergency situation.

At the first stage of development the system is stable functioning (tA). Over time, the system evolves in the direction of reducing its stability, that is, the system passes from state A to state B. At the same time, the trajectory AB reflects only the general development trend caused by the concept of admissible risk and the steady growth of the entropy of the system under study. The transformation of the system on the plot of the graph AB is due to the concentration of effects contributing to the emergence of an emergency situation.

Point B of the graph (bifurcation point) has an extremely important value in the development of the system, because it is in it there is a random choice between the vector of stability and the state of catastrophic development of the system [10]. Evolution at this point can manifest as a "+" sign, or with a "-" sign. With the growth of the "density" of negative factors, the system gradually loses its initial stability and goes into a catastrophic development mode, which is reflected in the curve of the aircraft's curve (the stage of the accident's injection).

The state of the system in T.C is characterized by the release of a significant part of the energy that goes into the further development of the accident scenario. The set of states of the system on the trajectory $\mathrm{CD}$ is usually called the extreme period or stage of the culmination.

After "dumping" of the main part of the energy, the system enters the decay period (the path section of DEI), characterized by a relative decrease in the degree of instability and the formation of new bifurcation points E1, E2 and E3, in which the system is capable of transitioning to other states. In the graph of Fig. 1, these states are respectively reflected by the trajectories E1F1, E2F2 and E3F3.

For example, the dynamics of the development of the system along the vector E1F1 translates it into a more stable state, but the state is still not stable enough. In this case, the active phase of the development of the system under investigation has not yet been completed and, taking into account external causes and circumstances, it is likely either to iterate the catastrophic development scenario, or a qualitatively new system will gradually balance its energy potential and move to the stability zone. It is also possible to form a scenario for the development of the system along the trajectory DE2 and beyond it E2F2. In this case, the system moves to the stability zone and its state can be regarded as metastable. And, finally, the evolution of the system along the trajectory DE3 and E3F3is possible. The system gradually returns to a more stable state, in comparison with the beginning of the development process, because the point $\mathrm{E} 3$ lies below point $\mathrm{A}$. 
Thus, the entire ABCDEiFi trajectory as a whole displays a graphic interpretation of the process of dynamic development of the system in the event of an accident. At the same time, in its development the system under investigation passes through 3 conditional phases, presented in Table 1.

Table 1. Phases of system development in case of accident.

\begin{tabular}{|c|c|c|}
\hline $\begin{array}{c}\text { Phase of } \\
\text { development } \\
\text { of the } \\
\text { system }\end{array}$ & \multicolumn{1}{|c|}{ Characteristics of the dynamics of the process } & The plot of the graph \\
\hline 1 & $\begin{array}{l}\text { The zone of stability, characterized by the growth of } \\
\text { negative factors and processes }\end{array}$ & AB \\
\hline 2 & $\begin{array}{l}\text { Transition from the phase of passive development to the } \\
\text { active phase of the development of the accident. The } \\
\text { characteristic point of the second phase is the bifurcation } \\
\text { point B. This phase is characterized by the presence of } \\
\text { many elements of the stable state of the system and } \\
\text { catastrophic development }\end{array}$ & $\begin{array}{l}\text { The zone of catastrophic development of the system with } \\
\text { the active growth of many elements of catastrophic } \\
\text { development and a sharp decrease in the set of elements of } \\
\text { sustainable development with the subsequent transition to } \\
\text { the region of stable states [10] }\end{array}$ \\
\hline 3
\end{tabular}

Graphical interpretation $\mathrm{ABC}$ trajectory allows carrying out a formalized assessment of the accident risk and using its value as a probabilistic characteristic of the degree of the possibility of the system transitioning to the emergency operation mode. From the graph in Fig. 1 it follows that the overall risk of a system transition to the active phase of an accident can be determined from expression

$$
R=\mathrm{P}_{A B} \mathrm{P}_{B C} \mathrm{P}_{C D}
$$

where $\mathrm{PAB}, \mathrm{PBC}, \mathrm{PCD}$ - is the mathematical probability of system transitions from state $A$ to state $B$, from $B$ to $C$, from $C$ to $D$, respectively.

In the presented interpretation, the magnitude of the risk of loss of stability of the system is defined as the product of the probability of a system transition from a stable state to a state of indifferent equilibrium and the probability of its transition from an equilibrium state to a zone of catastrophic development.

\section{Practical recommendations and conclusions}

Thus, the proposed formalized assessment of the physical indicators of the system's functionality, as well as the graphical-analytical model of the system development in the event of an accident, is of exceptional heuristic and methodological importance when deciding questions of safety management systems.

The approach of estimating the pre-emergency state of systems presented in the article, in our opinion, allows us to supplement the widely used in practice probabilistic approaches with elements of determinism and increases the efficiency of predicting system transitions to emergency situations.

The proposed graphical-analytical interpretation of the accident as a quasi-stationary process practically forms the direction of actions to ensure the stability of the system (on the branch of $\mathrm{AB}$ states) and forms prerequisites for the development of forecast models that assess the risk of accidents and the scenarios for their occurrence. It should be noted that such 
models must necessarily take into account the requirements of industrial safety, specific operating conditions of the system, its technical and economic capabilities. Under these conditions, dynamic models can become a methodological basis for the formation of a strategy for managing the security of systems.

\section{References}

1. V. M. Zababurin, Izv. universities. North-Caucasus. region. Techn. Science, 3, 94 (2014)

2. A. V. Frolov, V. M. Zababurin, Mater. International. scientific-practical. Conf. "White Nights-2010", 105 (2010)

3. V. M. Zababurin, Evaluation of pre-emergency state of ergotic systems / Technospheric security, reliability, quality, energy saving, 1, 14, 105 (2012)

4. V. M. Zababurin, Technospheric security, reliability, quality, energy saving: Mater. Int. scientific-practical. Conf., 1, XV, 105 (2013)

5. V.M. Zababurin, Mining Information and Analytical Bulletin, 12, 16 (2009)

6. U.R. Ashby, Introduction to Cybernetics (ComKniga, Moscow, 2006)

7. S.V. Belov, A.V. Ilnitskaya, A.F. Koziakov and others, Safety of life: Proc. for universities (Higher education, Moscow, 2005)

8. Yu. I. Medvedev, Lecture course. Part 2: Training allowance (Tomsk University, Tomsk, 2006)

9. V.P. Arnold, The theory of catastrophes (Nauka, Moscow, 1990)

10. A.Yu. Loskutov, A.S. Mikhaylov, Introduction to synergetics. Textbook. guide (Science, Moscow, 1990) 